Conclusions Modified-Delphi technique allowed for transparency surrounding consensus and estimated bundle element impact.

\section{IHI ID 14 RATIONAL OPIOID PRESCRIBING}

Adam Ackerman, L Scott Sussman, Deirdre Doyle, Christopher Zematis, Ryan Telford, Anesta Williams, Christine Day, Rockman Ferrigno, David Hajdasz, Bill Sotire, Catherine Fanning, Connie Cholewczynski. Yale New Haven Health, USA

\subsection{6/ihisciabs. 14}

Background At Yale New Haven Health System, our default route for delivering parenteral opioids has previously been the intravenous route, which can increase the risk of hypotension, undesired central nervous system effects and potentially risk of addiction. A multidisciplinary team identified opportunities to modify how opioids are prescribed and administered to reduce the risk of these adverse effects.

Objectives To reduce the number of IV opioid doses administered to adult inpatients, emergency department and observation patients by $25 \%$ through the adoption of a system-wide opioid prescribing standard of practice.

Methods The redesigned practice includes four main principles: EPIC Optimization: The removal of the default route of intravenous delivery of parenteral morphine, hydromorphone and fentanyl orders for adult patients. Education of providers to consider prescribing non-opioid pain medications and/or oral opioids first with subcutaneous delivery as the preferred parenteral route. Education of clinical nurses to consider subcutaneous delivery as the preferred route when parenteral opioids are required and to have a questioning attitude if opioids are routinely prescribed via intravenous delivery. Development of patient education material to provide information about the preferred route for administration of opioids as oral and subcutaneous.

Results Please see the attachment with the data analysis.
Conclusions Our data suggests these interventions were helpful in reducing exposure to IV opioids while still providing effective pain control to hospitalized adults. The work of the team allowed patients to experience consistent delivery of pain medication, better pain control with decreased unwanted side effects.

\section{IHI ID 15 DECREASING LOW-DOSE, RESCUE SEIZURE MEDICATION PRESCRIPTIONS IN NEUROLOGY CLINIC}

Shannon Yarosz, William Parker, Annika Gibson, Anup Patel. Nationwide Children's Hospital, USA

\subsection{6/ihisciabs. 15}

Background Children with seizures who are given incorrect dosing of rescue seizure medications may have a higher risk of emergency department visits, unplanned hospitalizations and even death. As such, it is critically important that children with seizures not only receive timely treatment but also treatment with the correct dose. However, due to a fear of complications, rescue medications are frequently under-dosed (low dose). Low dose treatment is often ineffective in halting seizures.

Objectives Decrease the percentage of low dose prescriptions for rescue seizure medications placed in a Neurology clinic from $3.5 \%$ to $1.5 \%$ by December 2018 and sustain for one year.

Methods We analyzed all rescue medication prescriptions from 2013-2015. We used 2015 data to determine a baseline rate of low-dose prescriptions, while using 2013-2015 data to identify the most commonly miss-prescribed medications. We developed a Key Driver Diagram and implemented the following interventions: Midazolam pre-filled syringe program, standardized dosing, electronic chart alerts, provider education, and pharmacy reviews of each low dose.

Abstract IHI ID 14 Table 1 Control charts and statistical tests: ANOVA comparison and mood test

\begin{tabular}{|c|c|c|c|c|}
\hline & IV Doses & Total MME & Pain Scores & LOS \\
\hline Control Chart Type & X Bar Chart & X Bar Chart & X Bar Chart & X Bar Chart \\
\hline Baseline Performance Mean & 1971.2 & 38409 & 4.68 & 4.67 \\
\hline Baseline Performance $95 \%$ Confidence Interval & $1940.3-2002.2$ & $37873-38945$ & $4.61-4.75$ & $4.57-4.75$ \\
\hline Current Performance Mean & 1150.1 & 28464 & 3.82 & 4.46 \\
\hline Current Performance $95 \%$ Confidence Interval & $1110.7-1189.4$ & $27860-29067$ & $3.79-3.85$ & $4.41-4.58$ \\
\hline Statistical Test & $\begin{array}{l}\text { ANOVA Comparison of } \\
\text { Means, } p=0.000\end{array}$ & $\begin{array}{l}\text { ANOVA Comparison of } \\
\text { Means, } p=0.000\end{array}$ & $\begin{array}{l}\text { ANOVA Comparison of } \\
\text { Means, } p=0.000\end{array}$ & $\begin{array}{l}\text { Moods Test Comparison of } \\
\text { Medians, } p=0.002\end{array}$ \\
\hline Method for Indicating Out of Control Signals & $\begin{array}{l}\text { Data points that meet ar } \\
1 \text { point }>3 \text { standard dev } \\
9 \text { points in a row on the } \\
6 \text { points in a row all inc } \\
14 \text { points in a row alterr }\end{array}$ & $\begin{array}{l}\text { following criteria are indi } \\
\text { om the center line } \\
\text { de of the center line } \\
\text { r decreasing } \\
\text { and down }\end{array}$ & a red dot on the control & \\
\hline
\end{tabular}




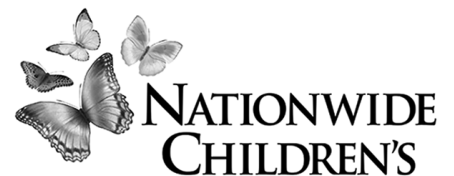

When your child needs a hospital, everything matters."
Decreasing Low-Dose Rescue Seizure Medication Prescriptions in Neurology Clinic Shannon Yarosz, PharmD ${ }^{1}$; William Parker, BS ${ }^{2}$; Annika Gibson, BS ${ }^{2}$; Anup Patel, MD ${ }^{1}$ Department of Pharmacy, Nationwide Children's Hospital, Columbus, Ohio, USA

Quality Improvement Services, Nationwide Children's Hospital, Columbus, Ohio, USA ${ }^{3}$ Department of Neurology, Nationwide Children's Hospital, Columbus, Ohio, USA

\section{Objective}

Decrease the percentage of low dose signed prescriptions, for rescue seizure medications placed in a Neurology clinic, from $3.5 \%$ to $1.5 \%$, by December 2018 and sustain for one year

\section{Background}

- As many as $30 \%$ of patients ultimately diagnosed as having epilepsy will initially present with status epilepticus, making it a common neurologic emergency ${ }^{1}$

- Current medical evidence suggests that there is a $20 \%$ mortality risk for those patients diagnosed with status epilepticus ${ }^{1}$

- Low dose rescue medication has been found to be a variable for high Emergency Department utilization ${ }^{2}$

- Patients who are given incorrect dosing of emergency seizure medications, or no emergency seizure medication dosing may have a higher risk of emergency departments visits and/or unplanned hospitalizations

- In 2017, the American Academy of Neurology and Child Neurology Society created a quality measure around this subject

\section{Methods}

- A multidisciplinary quality improvement (QI) team was established to conduct analysis of data for prescribed seizure rescue medication doses from January 2013 - December 2015

- Based on data analysis, the QI team identified areas of focus for improvement opportunities and developed the project objective

- The QI team developed key drivers and implemented interventions, such as midazolam prefilled syringe program with use of standardized dosing, creating electronic chart alerts and tools, monthly pharmacy review of all low dose prescriptions, and provider and nursing education

- An automated monthly report was created to monitor prescribed seizure rescue medication dosing compliance by providers

Breakdown of low dose orders by medication type

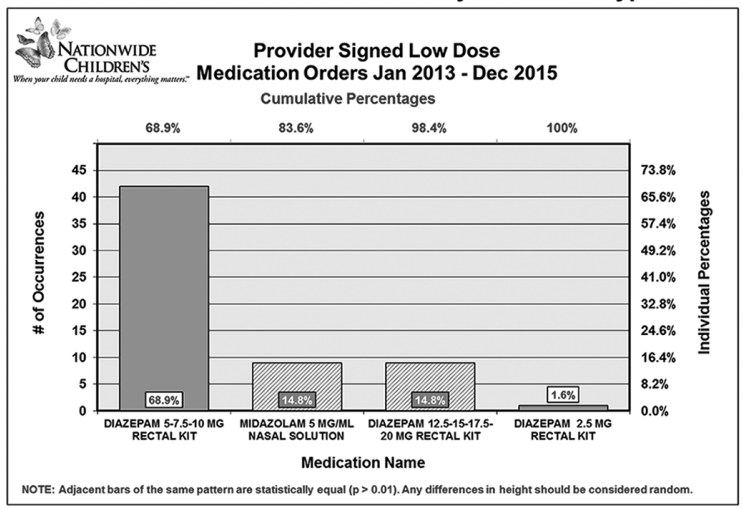

The Improvement Team
- Section Chief
- Nurses
- QI Medical Director
- Clinical Leader
- Neurology Pharmacist
- QI Coordinator
- Neurology Providers
- QI Data Analyst

\section{References}

1. Betjemann JP. Trends in Status Epilepticus-Related Hospitalizations and Mortality: Redefined in US Practice Over Time. JAMA Neurol. 2015 Jun;72(6):650-5.

2. Patel $A D$. Variables associated with emergency department and/or unplanned hospital utilization for children with epilepsy. Epilepsy and Behavior. 31 (2014) 172-175.

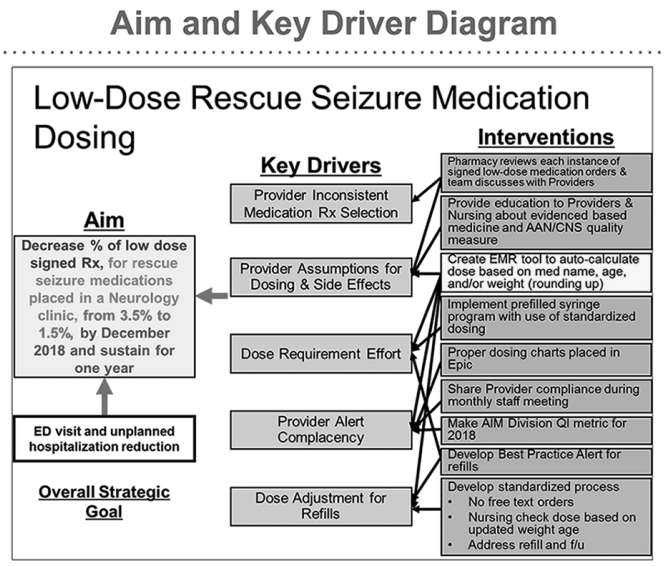

Results

- 2015 was used as the preliminary data baseline period with an average compliance rate of $3.5 \%$. From January 2016 - August 2018 , the team has decreased the non-compliance rate to an average of $1.6 \%$

- The p-chart below represents a total of 8,327 seizure rescue medication prescriptions signed by a provider from January 2015 - August 2018

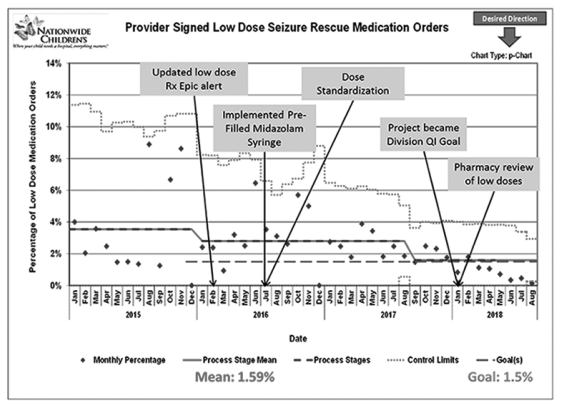

- Compliance of properly dosed Diazepam orders continues to be the largest area of opportunity. The p-chart below illustrates that no centerline shift or has occurred since the initial baseline average of $7.1 \%$ was established in 2015

- In comparison, low dosed and signed Midazolam orders occurred at an average rate of $0.33 \%$ in the same timeframe

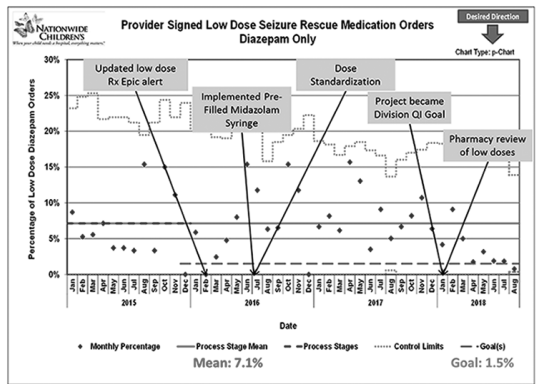

\section{Conclusion}

Using quality improvement methodologies, the team successfully and substantially decreased provider prescribed and signed low dose rescue medication orders by an average of $54 \%$ 
Results The most commonly identified low-dose medication was rectal Diazepam. Our baseline rate of low-dose prescriptions using data was $3.5 \%$. Interventions targeting efforts to eliminate low-dose prescribing resulted in a centerline shift from a baseline of $3.5 \%$ to $2.8 \%$ in January 2016. There was a second centerline shift to 1.59\% in September 2017.

Conclusions Using quality improvement methodologies, the team substantially decreased low dose rescue medication orders by an average of $54 \%$. We are currently developing a tool within our EMR to auto-calculate the correct rescue medication dose for each patient. \begin{tabular}{|l|l}
\hline IHI ID 16 & MOVING FROM PARALLEL TO SERIAL PREOPERATIVE
\end{tabular} PROCESS FLOW UTILIZING MULTIDISCIPLINARY BEDSIDE HANDOFFS IMPROVES PREOPERATIVE TASK COMPLETION

${ }^{1}$ Sean Josephs, ${ }^{2}$ Jennifer Raleigh, ${ }^{3}$ Leslie Wooten, ${ }^{2}$ Frank Adams, ${ }^{4}$ Jamie Chadwell, ${ }^{2}$ Alison Trammell, ${ }^{3}$ Lynne O'Brien, ${ }^{3}$ Andrea Merkel, ${ }^{2}$ Todd Osborne, ${ }^{4}$ Ron Wallmann, ${ }^{1}$ Alex Topala, 'Stuart Bertsch. 'University of Cincinnati College of Medicine, USA; ${ }^{2}$ UC Health University of Cincinnati Medical Center, USA; ${ }^{3}$ University of Cincinnati College of Nursing, USA; ${ }^{4}$ University of North Carolina, USA

10.1136/ihisciabs. 16

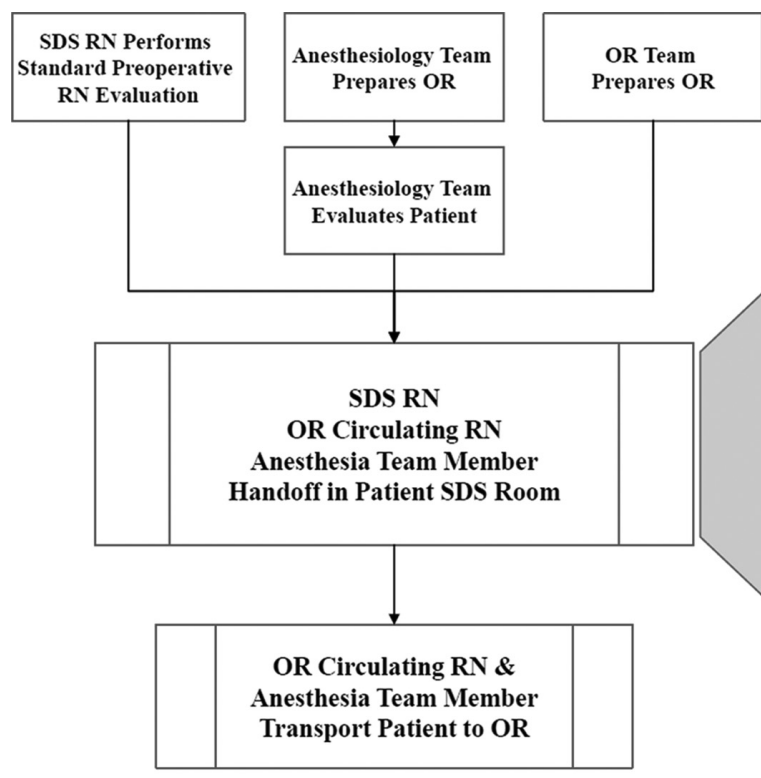

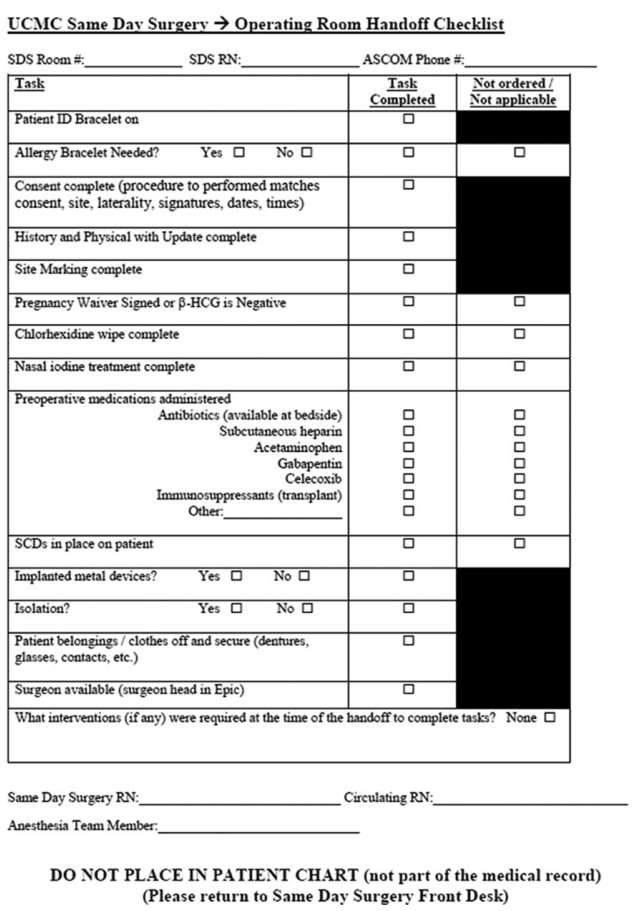

Abstract IHI ID 16 Figure 2 Days-between preoperative task completion failures

Individuals (XMR) chart depicting the days between preoperative task-completion failures that resulted in incident reports. The chart is annotated for important time points in the study. Special cause is illustrated by points/connectors in red and by points above the upper control limit. Dashed red line = upper control limit; Light blue line = Centerline depicting the mean for each value 